Известия НАН Армении, Физика, т.57, №1, с.125-130 (2022)

УДК 53.087 .9

DOI:10.54503/0002-3035-2022-57.1-125

\title{
РЕГИСТРАЦИЯ РАДИОСИГНАЛОВ МОЗГА И ИХ БИСПЕКТРАЛЬНЫЙ АНАЛИЗ
}

\author{
Г.Л. АЙВАЗЯН ${ }^{2}$, С.В. АНТОНЯН ${ }^{2}$, А.О. МАКАРЯН ${ }^{1}$, \\ Б.А. ОГАНЕСЯН ${ }^{1 *}$, Э.Р. СИВОЛЕНКО ${ }^{1}$, Г.А. ЦАТУРЯН ${ }^{2}$ \\ ${ }^{1}$ Ереванский государственный университет, Ереван, Армения \\ ${ }^{2} \mathrm{OOО} \mathrm{Нэшнл} \mathrm{Инструментс} \mathrm{АМ,} \mathrm{Ереван,} \mathrm{Армения}$ \\ *e-mail: Babken.hovhannisyan.96@gmail.com \\ (Поступила в редакцию 14 апреля 2021 г.)
}

С помощью зондовый антенны аппликатора зарегистрированы радиочастотные сигналы (РЧС) мозга человека. Предложен метод цифровой обработки регистрированных РЧС в среде LabVIEW применением спектрального и биспектрального анализа. В результате спектрального анализа выявлено, что человеческий мозг испускает РЧС в мегагерцовом диапазоне, а как свидетельствуют результаты биспектрального анализа, некоторые группы спектральных компонент РЧС мозга проявляют внутреннюю корреляцию. Результаты настоящих исследований могут быть полезны для выявления быстропротекающих процессов в мозге человека, для понимания природы этих процессов, а в результате для диагностики и лечения различных заболеваний человека, связанных с головным мозгом.

\section{1. Введение}

В настоящее время функциональная диагностика здоровья человека, в данном случае - наблюдения за активностью человеческого мозга, в большинстве случаев широко выполняется с применением электроэнцефалографа (ЭЭГ) $[1,2]$.

Это довольно чувствительный метод исследования функции коры головного мозга и глубинных мозговых структур. Электроэнцефалограф регистрирует колебания напряжения индуцированного на скальпе человека в результате ионного тока в нейронах головного мозга. Его чувствительность гораздо выше других методов исследований, в частности - позитронно-эмиссионной томографии (ПЭТ) и функциональной магнитно-резонансной томографии (ФМРТ). С помощью ЭЭГ можно выявить реакцию мозга на внешние раздражители, действующие на человека $[3,4]$. Однако, электроэнцефалограф регистрирует только низкочастотные сигналы. Введена классификация частот ЭЭГ по некоторым основным диапазонам, которым присвоены названия букв греческого алфавита: дельта (0.5-3 Гц), тета (4-8 Гц), альфа (8-13 Гц), бета (14-40 Гц) и гамма (больше 
40 Гц) [5]. Хотя ЭЭГ сигналы отражают состояние активности коры мозга, но не показывают информационных отношений между различными отделами мозга и не способны отразить деятельность высшей нервной системы человека: память, мышление, интеллект, процессы формирования речи и т.п. [6].

В ряде работ с помощью радиометрии подробно исследовано излучение биологических объектов, в том числе человеческого мозга $[5,7,8]$. Показано, что помимо сигналов ЭЭГ человеческой мозг испускает также электромагнитное излучение в СВЧ диапазоне.

В настоящей работе исследованы радиочастотные сигналы (РЧС), испускаемые мозгом человека и регистрируемые с помощью специального датчика (зондовой антенны-аппликатора). Зарегистрированные сигналы подвергались цифровой обработке в среде LabVIEW путем применения спектрального и биспектрального анализа. В результате спектрального анализа были обнаружены РЧС человеческого мозга в мегагерцовом диапазоне, а биспектральный анализ этих сигналов показал, что некоторые группы спектральных компонент РЧС мозга проявляют внутреннюю корреляцию.

\section{2. Регистрация и обработка РЧС мозга человека}

Для регистрации и статистической обработки РЧС была разработана система, блок схема которой приведена на рис.1. В качестве сенсора РЧС была использована специальная зондовая антенна-аппликатор, структурная схема которой изображена на Рис.1а.

Для проведения исследований были выбраны 15 пациентов-добровольцев. Исследования проводились следующим образом: к голове пациента была приложена антенна-аппликатор, сигнал с выхода которой по коаксиальному кабелю передавался на вход векторного трансивера (Vector Signal Tranciever) марки

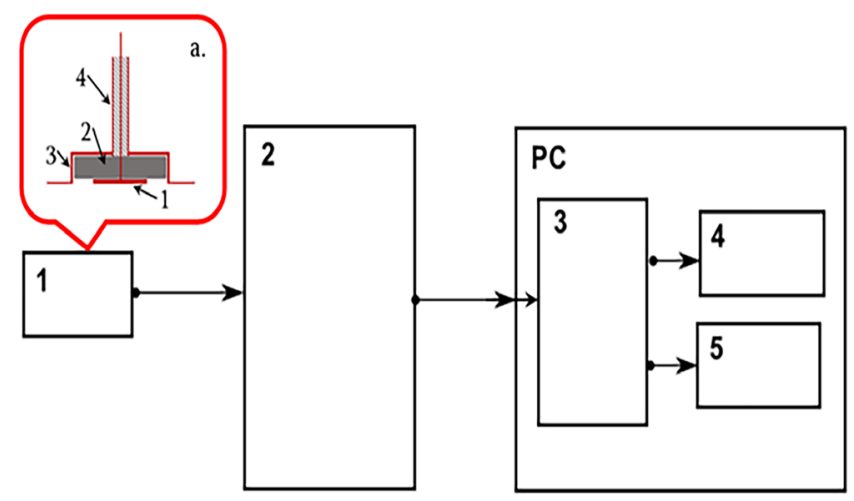

Рис.1. Блок схема системы регистрации и статистической обработки РЧС: 1 - антенна, 2 - VST (NI PXIe-5840), 3 - программный блок для предварительной обработки сигнала в среде LabVIEW, 4 - спектральный анализатор в среде LabVIEW, 5 - биспектральный анализатор в среде LabVIEW. (a) Структурная схема зондовой антенны-аппликатора: 1 - металлический электрод, 2 - изолятор, 3 - экранирующий электрод, 4 - коаксиальный кабель. 
NI PXIе-5646. После предварительного усиления и оцифровки сигнал передавался на компьютер для последующей обработки.

С помощью блока предварительной обработки 3 была отфильтрована фоновая составляющая сигнала, после чего сигнал был подвергнут спектральному и биспектральному анализу.

Спектральный анализ проводился в блоке 4, соответственно формуле дискретного преобразования Фурье [9]:

$$
S\left(f_{n}\right)=\frac{1}{N} \sum_{k=0}^{N-1} s\left(t_{k}\right) \exp \left(-j 2 \pi f_{n} t_{k}\right),
$$

где $s\left(t_{k}\right)$ - отсчетные значения сигнала, взятые в моменты времени $t_{k}=k T_{a} / N$, $T_{a}=N T_{d}$ - общая длительность сигнала (время анализа), где $N$ количество отсчетов, а $T_{d}$ - период дискретизации, $f_{n}=n / T_{a}$ - частота соответствующего спектрального компонента, $j=\sqrt{-1}$.

Для цифровой обработки сигнала удобнее представить (1) через индексы частотного и временного отсчетов $n$ и $k$ :

$$
S(n)=\frac{1}{N} \sum_{k=0}^{N-1} s(k) \exp \left(-\frac{j 2 \pi n k}{N}\right) .
$$

Для обеспечения статистической точности, сигнал, полученный от каждого пациента, регистрировался в течение достаточно большого времени ( 10 ми.) в изолированной от внешних воздействий комнате.

Временная форма радиосигнала мозга одного из пациентов, находящегося в спокойном состоянии представлена на Рис.2a, а на Рис.2b приведены результаты спектрального анализа этого сигнала по алгоритму дискретного преобразования Фурье.

Спектральный анализ регистрированного сигнала показывает, что вблизи 15 МГц есть излучение, которое отличается от фона. Однако это излучение не проявляло каких-либо особенностей, отличающих его от шума, которые были бы заметны на временной форме или на спектре.

Для выявления особенностей сигнала в среде LabVIEW был проведен биспектральный анализ сигнала в блоке 5 (см Рис.1), что позволило не только правильно описать статистические характеристики наблюдаемого процесса, но и определить наличие корреляционных связей спектральных компонент в наблюдаемом процессе [10].

Согласно определению (см. [10]) биспектр (биспектральная плотность) это двумерное преобразование Фурье тройной автокорреляционной функции, которую при дискретном преобразовании Фурье можно записать в следующем виде:

$$
\dot{B}_{S}(m, n)=\sum_{p=-N+1}^{N-1} \sum_{q=-N+1}^{N-1} R_{S}(p, q) \exp [-2 \pi j(m p+n q) / N],
$$



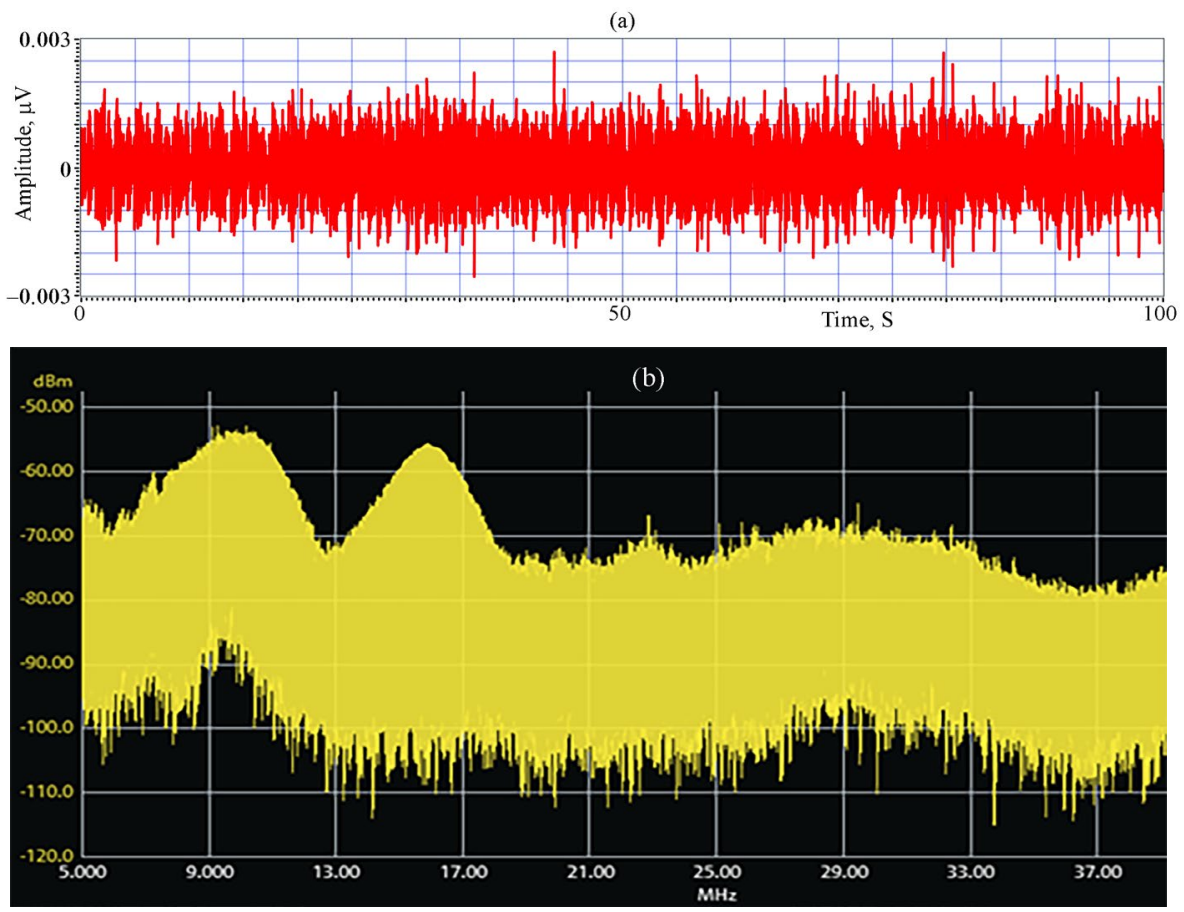

Рис.2. (а) Временная форма радиосигнала мозга пациента, регистрированного с помощью зондовой антенны-аппликатора, (b) спектр сигнала мозга.

где $m$ и $n$ - частотные индексы, $R_{x}(p, q)$ - тройная автокорреляционная функция:

$$
R_{s}(p, q)=\left\langle\sum_{k=0}^{N-1}\left[s^{(i)}(k)-E\right] \cdot\left[s^{(i)}(k+p)-E\right] \cdot\left[s^{(i)}(k+q)-E\right]\right\rangle,
$$

$p$ и $q$ - индексы отсчета дискретного временного сдвига, $k$ - индекс временного отсчета, $s^{(i)}(k)$ - это $i$-я реализации случайного сигнала, а

$$
E=\left\langle\frac{1}{N} \sum_{k=0}^{N-1} s^{(i)}(k)\right\rangle
$$

есть статистическое среднее сигнала по ансамблю реализаций.

При отсутствии постоянной составляющей сигнала, основываясь на (3) и (4), биспектр может быть выражен через спектры реализаций случайного сигнала $\dot{S}^{(i)}(m)[10]$ :

$$
\dot{B}_{S}(m, n)=\left\langle\dot{S}^{(i)}(m) \dot{S}^{(i)}(n) \dot{S}^{*(i)}(m+n)\right\rangle=\left\langle\dot{S}^{(i)}(m) \dot{S}^{(i)}(n) \dot{S}^{(i)}(-m-n)\right\rangle,
$$

т.е. биспектр - это среднее статистическое значение тройного произведения спектров на частотах $m, n$ и комплексно сопряженного спектра на частоте $m+n$, которое характеризует взаимные корреляционные отношения между спектральными компонентами процесса на этих трех частотах.

Результаты биспектрального анализа сигнала, спектр которого изображен на Рис.2, представлены на Рис.3. 


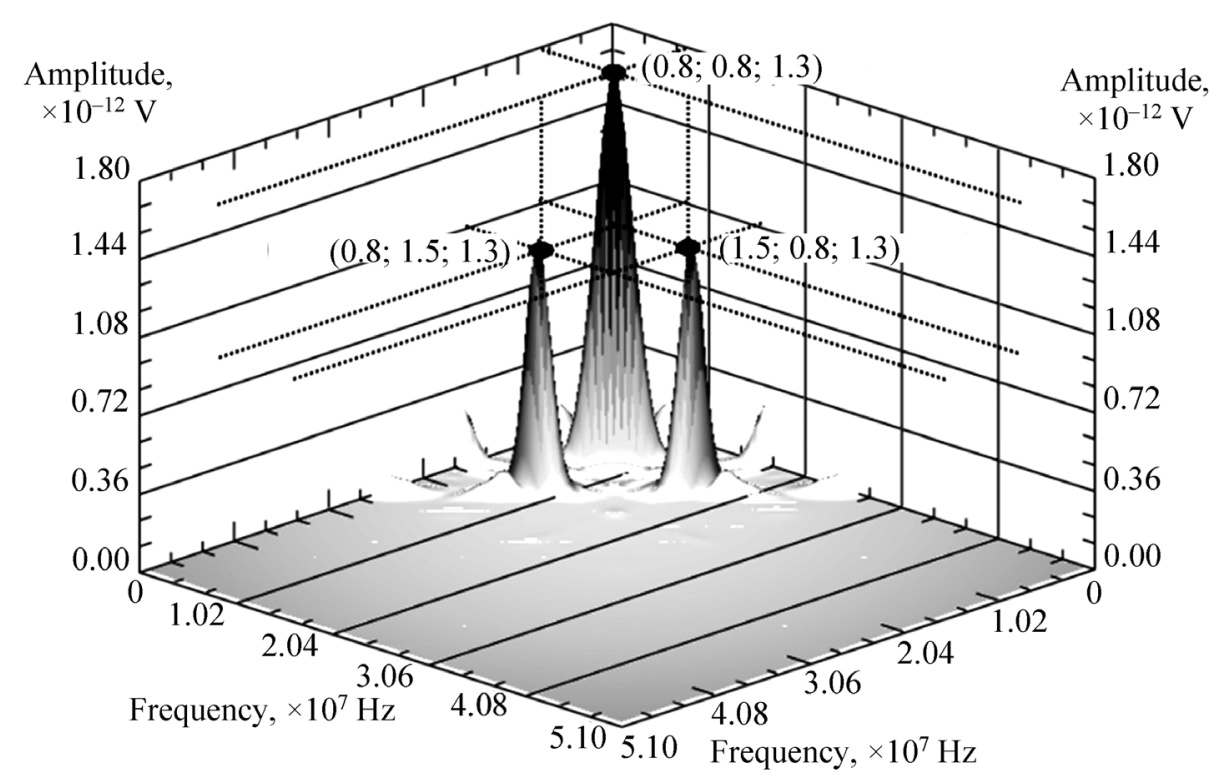

Рис.3. Биспектр радиосигнала мозга человека.

На рисунке отчетливо видны области частот, где биспектральная плотность регистрированного сигнала мозга человека существенно отличается от фонового значения. Это означает, что спектральные компоненты сигнала на этих частотах $\left(f_{1} \approx 8\right.$ МГц и $f_{2} \approx 15$ МГц) взаимно-коррелированы.

На графике видны также другие отличающиеся от фона спектральные компоненты ( $f_{3} \approx 8$ МГц и $f_{4} \approx 18$ МГц и т.д.), однако они относительно слабо коррелированы между собой.

Сравнительный анализ спектров и биспектров 15-и пациентов-добровольцев свидетельствует о том, что, в спектральном составе радиосигнала мозга человека есть коррелированные компоненты в мегагерцовом диапазоне. Частоты коррелированных компонентов у разных людей немного отличаются друг от друга, но они лежат вокруг одних и тех же значений (8 МГц, 15 МГц, 18 МГц и т.д.).

\section{3. Заключение}

Результаты настоящих исследований показывают, что человеческий мозг генерирует радиосигналы в мегагерцовой области частот, которые можно регистрировать с помощью зондовой антенны аппликатора. Как свидетельствуют результаты биспектрального анализа, некоторые группы спектральных компонент радиосигнала мозга проявляют внутреннюю корреляцию, при этом частоты коррелированных компонентов у разных людей отличаются друг от друга, однако они лежат вокруг одних и тех же значений. Результаты настоящих исследований могут быть полезны для выявления быстропротекающих процессов в мозге человека, для понимания природы этих процессов, а в результате для диагностики и лечения различных заболеваний человека, связанных с головным мозгом. 


\title{
ЛИТЕРАТУРА
}

1. A. Maturana-Candelas et al. Frontiers in Computational Neuroscience, 24, 70 (2020).

2. C. Gómez. 39th Annual International Conference of the IEEE Engineering in Medicine and Biology Society (EMBC), Jeju, Korea (South), 422 (2017).

3. C. Babiloni, F. Babiloni, F. Carducci, F. Cincotti, G. Cocozza, C. Del Percio, D.V. Moretti, P.M. Rossini, H. Cortical. NeuroImage, 17, 559 (2002).

4. B. Roth. Electroencephalography \& Clinical Neurophysiology, 13, 395 (2016).

5. Л.И. Брусиловский, А.С. Брюховецкий, С.П. Кожин, П.Г.Серафимович, А.В. Никоноров. Журнал радиоэлектроники, № 2, 1684 (2020).

6. P.A. Abhang, B.W. Gawali, S.C. Mehrotra. Technological Basics of EEG Recording and Operation of Apparatus, Academic Press, 2016.

7. J.W. Hand, G.M.J. Van Leeuwen, S. Mizushina. Physics in Medicine and Biology, 46, 1885 (2001).

8. K. Maruyama. IEEE Transactions on Microwave Theory and Techniques, 48, 2141, (2000).

9. С.И. Баскаков, Радиотехнические цепи и сигналы, Москва, Высшая школа, 2000.

10. А.А. Зеленский, В.Ф. Кравченко, В.В. Павликов, А.В. Тоцкий, Биспектральный анализ в задачах цифровой обработки сигналов, Физические основы приборостроения, 2(3), 4, (2013).

\section{REGISTRATION OF BRAIN RADIO SIGNALS AND ITS BISPECTRAL ANALYSIS}

\author{
H.L. AYVAZYAN, S.V. ANTONYAN, A.O. MAKARYAN, \\ B.A. HOVHANNISYAN, E.R. SIVOLENKO, G.A. TSATURYAN
}

Radio frequency signals (RFS) of the human brain are recorded using the probe antenna applicator. A method for digital processing of recorded RFS in the LabVIEW environment using spectral and bispectral analysis is proposed. As a result of spectral analysis, it was revealed that the human brain emits RFS in the megahertz range, and as evidenced by the results of bispectral analysis, some groups of spectral components of brain RFS exhibit an internal correlation. The results of these studies can be useful for identifying fast processes in the human brain, for understanding the nature of these processes, and, as a result, for the diagnosis and treatment of various human diseases associated with the brain. 